\title{
EXPLICIT COMMUNICATION OF GEOMETRIC DESIGN INTENT IN CAD: EVALUATING ANNOTATED MODELS IN THE CONTEXT OF REUSABILITY
}

\author{
Jorge Camba \\ Eng. Design Graphics \\ Texas A\&M University \\ College Station, TX, USA \\ Email: camba@tamu.edu
}

\author{
Manuel Contero \\ I3BH. Universitat \\ Politècnica de València \\ València, Spain, 46010 \\ Email: mcontero@upv.es
}

\author{
Jeffrey Otey \\ Eng. Design Graphics \\ Texas A\&M University \\ College Station, TX, USA \\ Email: j-otey@tamu.edu
}

\author{
Pedro Company \\ Inst. of New Imaging \\ Technologies. Univ. Jaume I. \\ Castelló, Spain, 12071 \\ Email: pcompany@uji.es
}

\begin{abstract}
CAD model reusability is largely determined by a proper communication of design intent, which is usually expressed implicitly within the model. Recent studies have suggested the use of $3 D$ annotations as a method to embed design information in the model's geometry and make part of the design knowledge explicitly available. In this paper, we evaluate the effectiveness of this method and analyze its impact in model alteration tasks. Our goal is to determine whether annotated models provide significant benefits when performing activities that require a direct manipulation of the geometry. We present the results of a study that measured user performance in two scenarios. First, we tested whether annotations are helpful when inadequate modeling assumptions can be made by designers. Second, we evaluated annotations as tools to communicate design decisions to select the most appropriate solution to a challenge when multiple options are available. In both cases, results show statistically significant benefits of annotated models, suggesting the use of this technique as a valuable approach to improve design intent communication.
\end{abstract}

\section{INTRODUCTION}

CAD model reuse is a key issue to leverage current commercial history-based parametric CAD systems. The degree of flexibility and the ease of alteration of a CAD model strongly depend on the modeling methodology rather than the technology. In the context of the Model-Based Enterprise (MBE), an efficient use of 3D CAD models determines the benefits of a successful implementation. In theory, modern mechanical CAD systems allow designers and engineers to reuse CAD elements throughout different stages of the product lifecycle, both as templates for new product configurations that are similar in function or shape and as a starting point for new product developments, thus accelerating the design process and increasing productivity. However, it has been shown that CAD model reusability largely depends on a proper definition and communication of the geometric design intent, which are usually expressed implicitly within the CAD model. In feature-based CAD packages, the modeling strategy and the parent-child relations established among the features of the model are conveyed implicitly via the design tree or history tree. Implicit definition of design intent typically requires a thorough examination of the model structure and a detailed understanding of the modeling strategy prior to performing any alteration to the geometry, which often translates to considerable amounts of manipulation time and effort, even when the required alterations are minor. This is especially true for models being reworked by designers that are not the original creators of the models [1]. The inability to understand and modify existing CAD models negatively affects reusability and hinders the collaborative design process.

Recently, some authors have suggested the use of model annotations as a mechanism to incorporate design information into the model's geometry and make part of the design knowledge explicitly available. In this paper, we present the results of two experiments that evaluate the effectiveness of 3D annotation techniques as a tool to express and communicate design intent information. Our goal is to determine whether annotated models have a significant impact over non-annotated models in activities that involve model alteration and reusability.

We begin by reviewing previous work in three relevant areas: CAD reusability (and how it relates to model alteration), design intent communication, and annotations in engineering design. Next, we describe the experimental procedure and how annotations were used in the study. Finally, we present the statistical analysis and discuss our findings.

\section{RELATED WORK}

\section{CAD Model Reusability}

Today's highly globalized markets are forcing engineering design and manufacturing firms to constantly increase performance and reduce development times and costs by delivering products that meet ever increasing customers' expectations with minimum delays. To guarantee competitiveness, this situation naturally demands the implementation of new methods and processes [2]. For example, traditional product development models are progressively being replaced by distributed and concurrent approaches, and the Model-Based Enterprise (MBE) concept is gradually gaining more ground.

In the context of distributed methodologies, CAD models have become essential assets that can be shared among designers, manufacturers, suppliers, and other stakeholders throughout the different stages of the product lifecycle [3-5]. In addition to their 
classic advantages such as improvement of product quality [6] and technical communication [7, 8], and reduction of product development times [9], CAD models provide even greater benefits when combined with Product Data Management (PDM) systems [10], as they become facilitators of the concurrent development that is possible in distributed environments [11].

According to many authors $[4,12,13]$, an important success factor in new engineering design and development methodologies is the ability to apply knowledge obtained in previous design processes to new designs, which directly translates to CAD model reusability. As noted by Iyer et al. [13], citing Ullman [12], the majority of design problems found in industry involve the application of previous knowledge and the redesign of existing products. Today, the high pressure put on enterprises for optimizing and accelerating product development processes is giving design reuse a crucial role in industrial environments.

There is evidence of the necessity of effective knowledge management methods for establishing efficient design and reutilization processes. Bodein et al. [14] reported that reusability of existing models was a critical factor when determining CAD modeling strategies in the automotive industry. According to an industry study by the Aberdeen Group [4], significant time and cost savings were reported in cases of companies reusing design elements. Furthermore, all engineering organizations surveyed in the study report the reuse of existing designs at some level, but the top performers intentionally dedicate resources and deploy methods and technologies to capitalize on reusability.

There are, however, important problems that need to be overcome to effectively implement design reuse. As described by the same study [4], the obstacles related to CAD model reuse are the same obstacles that largely impede design reuse. These challenges as well as the procedures that companies are currently following to mitigate them are shown in Table 1.

Table 1. Challenges and responses to CAD model reusability (adapted from [4])

\begin{tabular}{|ll|l|}
\hline \multicolumn{1}{|c|}{ CHALLENGE } & \multicolumn{1}{c|}{ PROCEDURE } \\
\hline $\begin{array}{l}\text { 1. } \\
\text { Model modification requires } \\
\text { expert CAD knowledge }\end{array}$ & Train users to increase CAD skills \\
\hline 2. & $\begin{array}{l}\text { Models are inflexible and fail } \\
\text { after changes }\end{array}$ & $\begin{array}{l}\text { Design for wide range of } \\
\text { modifications }\end{array}$ \\
\hline 3. & $\begin{array}{l}\text { Users cannot find models to } \\
\text { reuse }\end{array}$ & $\begin{array}{l}\text { Centralize design data in library } \\
\text { accessible structure }\end{array}$ \\
\hline 4. & $\begin{array}{l}\text { Only original designer can } \\
\text { change models successfully }\end{array}$ & $\begin{array}{l}\text { Detail design information in } \\
\text { model }\end{array}$ \\
\hline
\end{tabular}

We can clearly see the relationship between challenges 1 and 2, as they are both involved in the creation of better designed models. CAD users need to be skilled and trained in CAD methods, tools, and technology in order to develop models that are reusable and easy to maintain. Challenge 3 can be categorized as a data management problem that requires effective tools and information management mechanisms such as Product Lifecycle Management systems (PLM) to be in place. Finally, challenge 4 demands formal methods to integrate various types of design information (Geometric Dimensioning and Tolerancing, material specifications, manufacturing instructions, etc) within the CAD model. The development of Digital Product definition standards such as ASME Y14.41[15] and ISO 16792 [16] has formalized the way some product information is presented in a 3D model, which has encouraged many companies to migrate their development processes to the Model-Based Enterprise concept.

However, to ensure an effective CAD reusability it is necessary that users that interact with a particular model understand the reasons and rationale behind the modeling decisions. They need to know how and why the model was created in a specific manner, i.e., they need to understand its design intent $[17,18]$.

\section{Design Intent Communication}

It is difficult to find a generic definition of the term "design intent” in the technical literature. In fact, different definitions have been proposed [19-24]. An extensive survey was conducted by Iyer and Mills [17] that identified elements in a number of definitions that are common to all interpretations of design intent. The authors used this information to provide their own comprehensive definition in the domain of 2D CAD: "Design intent contained in legacy CAD is the insight into the design variables (design objectives, constraints, alternatives, evolution, guidelines, manufacturing instructions and standards) implicit in the structural, semantic and practical relationships between the geometric, material, dimensional and textual entities present in the CAD representation.” [17].

Despite a lack of consensus on the exact meaning, there is agreement on the importance of design intent and the advantages of an explicit representation. According to Pena-Mora et al. [25], some of these advantages include:

- Changes in complex projects require certain design decisions to be modified during the development process. When the justifications defined during the initial stages are lost, they need to be recreated, which has a negative impact on project costs and development times. The ability to store, process, and retrieve this information can significantly improve productivity.

- When design intent information is represented explicitly and is easily available for review, the overall quality of the product increases.

- Explicit representation of design intent leads to a more intelligent use of resources and knowledge.

- Efficient communication of design intent is essential for integrating solutions and transferring design knowledge. 
Despite those advantages, it has not yet been solved. In the same survey mentioned earlier [17], the authors classify the capture, representation, and retrieval of design intent as open issues and suggest avenues for future research.

Although significant advances in the area of Model-Based Definition have been accomplished (such as the definition of standards and the development of Product and Manufacturing Information (PMI) modules, it is still difficult to represent design intent information explicitly within the model using these tools.

PMI modules represent practical implementations of current Digital Product Definition standards such as ASME Y14.41[15] and ISO 16792 [16] are usually available in modern CAD systems. PMI tools allow users to incorporate product definition information such as Geometric Dimensioning and Tolerancing (GD\&T), 3D textual annotations, surface finish and material specifications to different aspects of the CAD model, as shown in Figure 1.

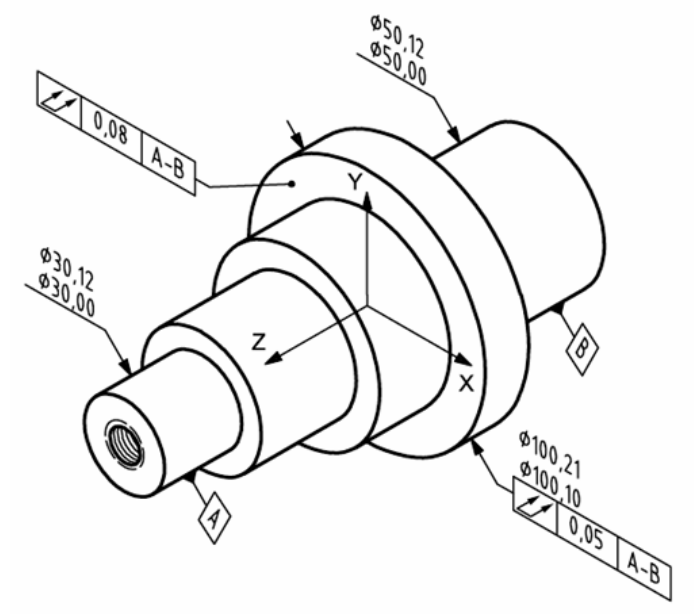

Figure 1. ANNOTATED EXAMPLE FROM ISO 16792:2006

To sum up, the ultimate goal of a product's Model-Based Definition (MBD), a dataset that comprises the model's geometry and annotations that specify life cycle support data, is to provide a complete representation and definition of a product without the need for additional documents. Representing and managing design intent information, however, has proven to be a difficult task. Recent studies have suggested the use of 3D annotations as a method to carry design intent information [26-29]. In this paper, we evaluate the effectiveness of this approach and analyze its impact in model alteration tasks.

\section{Annotations in Computer-Aided Design}

In the domain of 3D CAD, an annotation (also called model-based annotation or 3D annotation) is defined as a block of information that is linked to a specific aspect of a CAD model to provide additional details [30]. 3D annotations are normally used as pointers to direct the attention of the designers to a specific part of the model. They are usually inserted as textual notes or comments, but they can also adopt other forms such as images, audio, hyperlinks, etc.

Annotations can be classified in a variety of ways. Ding et al. [30] suggested six major categories of annotations based on audience, targeted media, rendering system, usage and function, representation, and storage location. In the context of engineering design, the significance of annotations was stated by Bracewell and Wallace [31] and Boujut and Dugdale [32], who emphasized the role of annotations as mechanisms to capture the rationale behind design decisions and to mediate interactions between designers. Nevertheless, they admitted these roles to be poorly addressed by current industrial tools.

Active research has been done to determine the suitability of 3D annotations to carry design information [28, 29, 32, 33]. Patel et al. [34] suggested supplementing CAD models with information that is relevant to the product lifecycle (such as design rationale, context, views, etc). Li et al. [35] recommended a unification of the representation of annotations to make them independent from specific platforms and systems. A number of prototypes have also been developed [27, 29, 32].

There are, however, a number of challenges to using 3D annotations to carry design knowledge. Some of these challenges are related to the technology, such as defining efficient internal structures and data types for the annotations or developing an interface or system to enter and retrieve the information [30]. Other challenges involve user interaction, such as the representation of the annotation content (what needs to be included in the annotation and in what form, so the information is communicated effectively) or the visualization of heavily annotated models (the problem of visual clutter and annotation overload). For our research, we conducted two experiments to analyze user interaction with CAD models that incorporate design information in the form of annotations. Our objective is to determine empirically the validity of $3 \mathrm{D}$ annotations as mechanisms to enhance model definition. In the following sections, we will describe the experimental procedure and present our findings.

\section{EXPERIMENTAL SETUP AND PROCEDURE}

The basis of our study is the application and use of 3D annotations in history-based parametric modeling processes. Our goal is to determine whether annotated models provide a significant benefit over non-annotated models in terms of reusability, specifically when performing tasks that require design alterations or manipulation of the model's geometry. 
We conducted two separate studies with a total of 104 volunteers. All participants had previous experience in engineering design graphics and parametric solid modeling, particularly using the CAD package SolidWorks ${ }^{\circledR}$. The experiments were conducted in a computer laboratory environment, where participants were equipped with a workstation and the modeling software. The first experiment was aimed at model alteration activities, whereas the second one focused on design tasks. For both experiments, participants were randomly divided into two groups. One group served as the control group (participants used non-annotated models to complete the tasks) and the other served as the experimental group (participants used models that were previously annotated with relevant design information by a member of the research team).

\section{Study 1: Geometry Modification}

Our first study was intended to obtain new insights on CAD modeling strategies and understand whether annotations can be added to a CAD model to communicate specific design intent information, so it is easier for designers to select the most appropriate modeling procedure when certain alterations need to be performed. In particular, we described a scenario where designers run the risk of making inadequate assumptions about the modeling process if design information is not explicitly defined.

We prepared an experimental activity involving a number of alterations to an existing parametric CAD model. The activity was presented as a series of questions in a classic test format, using an online testing tool. Each alteration in the sequence was presented as a separate question, requiring participants to submit their modified CAD model for each question. In order to accurately analyze the modeling process followed by participants for the entire sequence, questions were displayed one at a time and backtracking was intentionally disabled. Therefore, participants were forced to submit a CAD model for every question before moving to the next, and were not allowed to change the answer to a question that had already been submitted.

Participants were randomly divided into two groups of 52. The first one was a control group, whereas the second one served as the experimental group. All participants in both groups received the same CAD model in terms of geometry and modeling strategy. However, an annotation indicating not to assume symmetrical arms was added to the model provided to the experimental group. The initial CAD model used in this study is shown in Figure 2. Dimensions have been omitted for clarity.

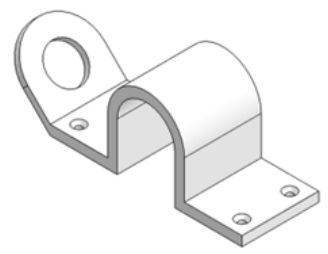

Non-annotated model (Control Group)

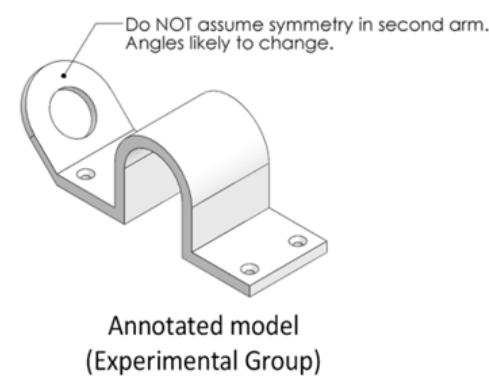

Figure 2. INITIAL MODEL PROVIDED TO PARTICIPANTS IN STUDY 1

The model was introduced as a design that had to be modified to meet new design requirements, so it could be reused as part of a different product or assembly. Neither user group was mentioned the existence of the annotation, nor were they given further instructions as to how they were supposed to use the information.

For illustration purposes, the basic modeling steps involved in the creation of the initial model are shown in Figure 3. Each step is the result of applying a single modeling operation using the CAD package (Solidworks ${ }^{\circledR}$ in our case).

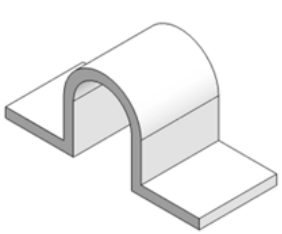

1. Base feature

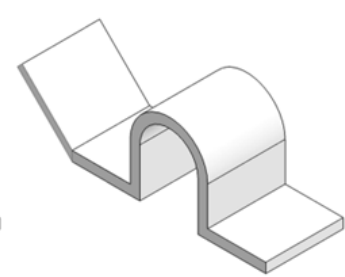

2. Extrude arm

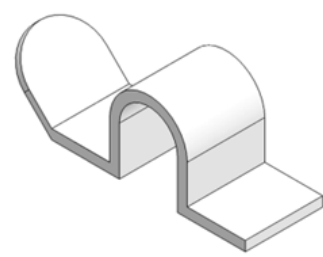

3. Round corners

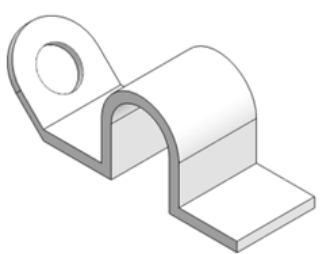

4. Cut hole on arm

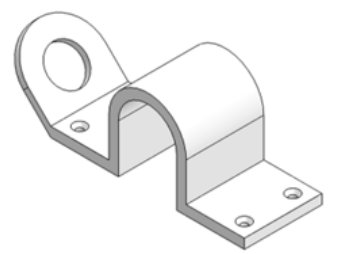

5. Cut machined holes on base

Figure 3. MODELING PROCESS OF ORIGINAL PART FOR STUDY 1 
The sequence of modifications requested to participants is shown in Figure 4. Alterations were presented one at a time. Therefore, participants were not allowed to see alteration $i$ until they completed alteration $i-1$.

The first modification task involves the addition of a second arm to the part. The new arm is identical to the existing one in both

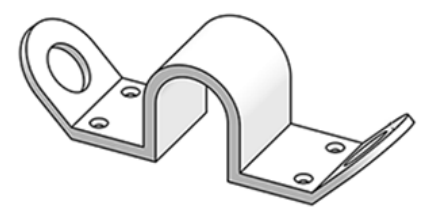

ALTERATION 1 (add second arm)

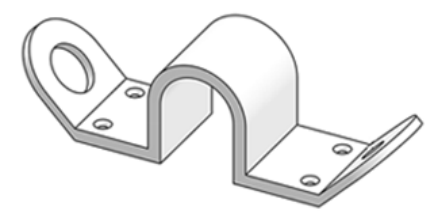

ALTERATION 2

(change hole diameter)

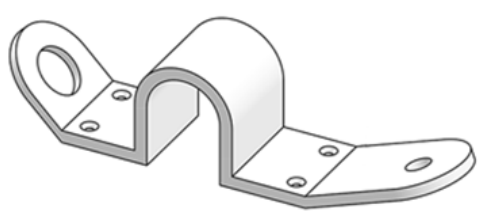

ALTERATION 3

(modify angle and size)

Figure 3. SEQUENCE OF ALTERATIONS REQUESTED IN STUDY 1

shape and size. There are, at least, two distinctive modeling approaches that can be followed: using symmetry tools to mirror all the features in the existing arm, or creating the second arm from scratch by defining a new sketch, extruding it, and cutting a new hole, i.e. repeating the process used to create the original arm (see Figure 5).

\section{APPROACH 1: MIRROR FEATURES}

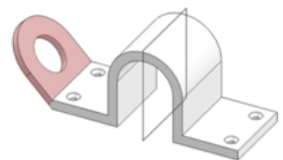

1. Select features to mirror (extrusion, fillet, and cut) and select mirror plane

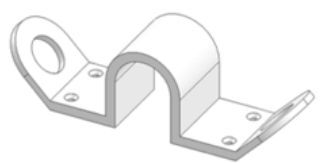

2. Mirror existing features

APPROACH 2: MODEL NEW FEATURES

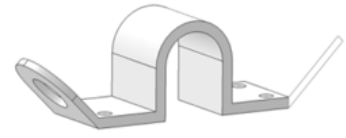

1. Create new sketch

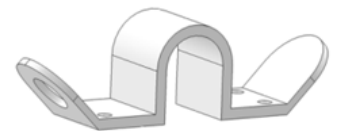

3. Fillet edges

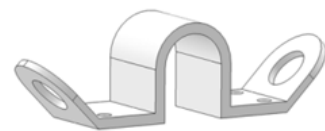

\section{Cut hole}

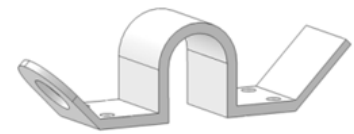

2. Extrude sketch

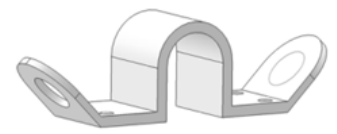

4. Create new sketch

Figure 5. TWO MODELING APPROACHES FOR "ALTERATION 1"

Instinctively, the first approach seems easier and faster than the second one, as it requires fewer steps and no features need to be modeled from scratch. The result is an efficient model as long as all features in both arms remain symmetrical in new variations of the part or other cases where the model needs to be reused. In other words, when selecting this approach, the designer is implicitly assuming that both arms are likely to remain symmetrical in future models, which is reasonable if "Alteration 1" is the only piece of information she possesses. Performing the first alteration to the model by creating the second arm from scratch may not seem like an efficient strategy, as it does require a number of additional steps. However, the geometry of each arm can be controlled and edited separately without affecting the other, should only one arm needed to be modified (which is precisely what happens in upcoming alterations). 
By analyzing the entire sequence, it seems clear that mirroring the existing arm (“approach 1") might not be the best approach, since both the angle and the length of the new arm will be modified in Alteration 3. In fact, if symmetry is assumed, alteration 3 will require the suppression or deletion of all mirrored features and the creation of the new arm from scratch (which is precisely "approach 2”). If we were using a more sophisticated model with a greater number of features that depended on the new arm, performing alteration 3 after mirroring would likely cause major rebuild errors and require a significant amount of time and effort to fix. Since none of this information is available to participants in the control group at the time they have to make a decision about Alteration 1 , we hypothesized that:

- If using the non-annotated model, the majority of participants will assume symmetry and use mirror tools to perform Alteration 1.

- If using the model that was previously annotated with relevant design information, the majority of participants will create the second arm by modeling the new features from scratch.

We define the modeling approach as the independent categorical variable $\mathrm{X}_{1}$, with values as shown in Table 2 . We note that, as previously discussed, in some cases during the experiment, as soon as alteration 2 was presented, some participants realized the need to control the two arms separately and decided to re-model the arm from scratch. This behavior is listed as “Approach 3 ” in table 2.

\begin{tabular}{|c|lll|}
\hline \multirow{2}{*}{$\begin{array}{c}\text { Values of } \\
X_{1}\end{array}$} & \multicolumn{3}{|c|}{ Description of the modeling approach } \\
\cline { 2 - 4 } & Alteration 1 & \multicolumn{1}{c|}{ Alteration 2 } & \multicolumn{1}{c|}{ Alteration 3 } \\
\hline $\begin{array}{c}\text { Approach } \\
1\end{array}$ & Mirror arm & $\begin{array}{l}\text { Edit previous } \\
\text { mirror and } \\
\text { create new hole }\end{array}$ & $\begin{array}{l}\text { Delete previous } \\
\text { mirror and re-model } \\
\text { arm. }\end{array}$ \\
\hline $\begin{array}{c}\text { Approach } \\
2\end{array}$ & $\begin{array}{l}\text { Model new } \\
\text { arm from } \\
\text { scratch }\end{array}$ & $\begin{array}{l}\text { Edit circle in } \\
\text { sketch and } \\
\text { update feature }\end{array}$ & $\begin{array}{l}\text { Change dimensions } \\
\text { of previous sketch } \\
\text { and update feature }\end{array}$ \\
\hline $\begin{array}{c}\text { Approach } \\
3\end{array}$ & Mirror arm & $\begin{array}{l}\text { Delete previous } \\
\text { mirror and re- } \\
\text { model arm from } \\
\text { scratch }\end{array}$ & $\begin{array}{l}\text { Change dimensions } \\
\text { of previous sketch } \\
\text { and update feature }\end{array}$ \\
\hline
\end{tabular}

The results of our study are shown in Table 3 and Figures 6 and 7.

TABLE 3. ACTUAL AND EXPECTED VALUES FOR STUDY 1

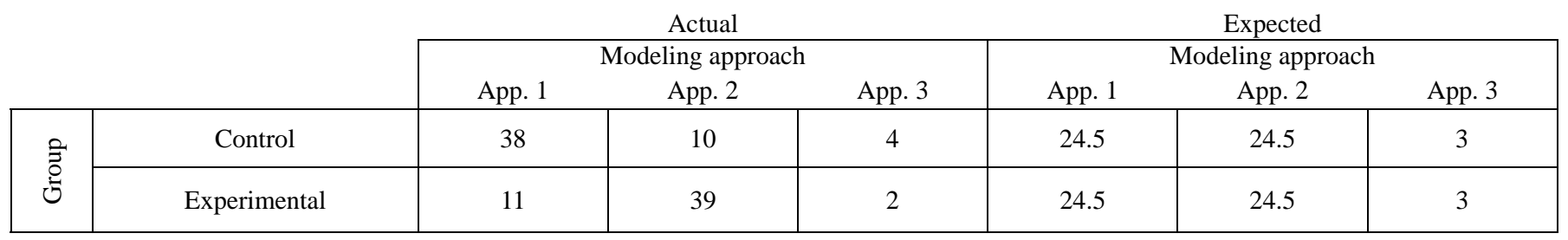

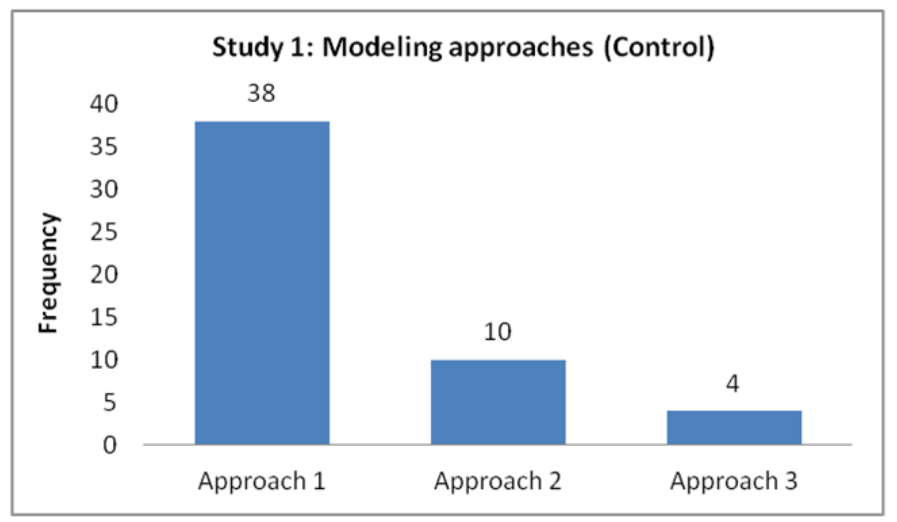

Figure 4. ACTUAL VALUES FOR STUDY 1 (CONTROL GROUP)

Due to the nature of our data, we performed a Chi-Square Test to examine the difference in modeling approaches between the control (non-annotated model) and experimental (annotated model) groups. Based on the resulting $\mathrm{p}$-value $(\mathrm{p}<0.001)$, we can conclude 
that there is a statistically significant difference between the experimental and control groups. Even with no prior warnings, the design information provided as an annotation contributes substantially to the selection of an efficient modeling approach.

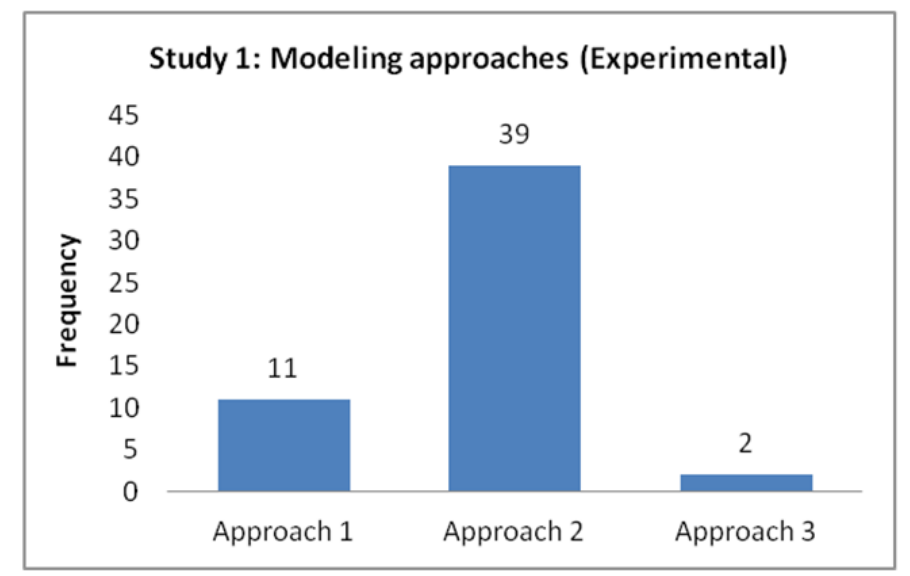

Figure 5. ACTUAL VALUES FOR STUDY 1 (EXPERIMENTAL GROUP)

\section{Study 2: Design Task}

The goal of our second experiment is to determine whether annotations are a valuable tool to communicate requirements, constraints, and modeling considerations in situations where design decisions must be made. Unlike the previous study, where participants performed a series of alterations to an existing model, in this experiment participants were required to find a solution to a design challenge. Multiple solutions to the problem were available.

The models provided to participants are shown in Figure 8. Dimensions have been omitted for clarity. Once again, participants in the experimental group received a version of the model that was previously annotated with relevant design information by a member of the research team.

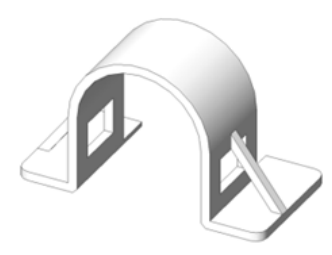

Non-annotated model (Control Group)

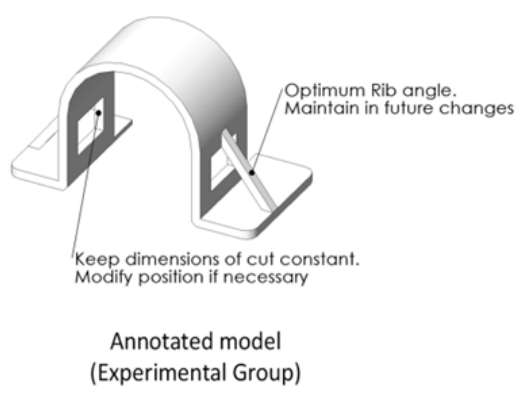

Figure 6. MODEL PROVIDED TO PARTICIPANTS IN STUDY 2

As a first step, participants were asked to increase the dimensions of the side ribs, from $4 \mathrm{~mm}$ to $5 \mathrm{~mm}$. This alteration is intentionally prepared to cause an unwanted effect in the model, as shown in Figure 9. Participants were then challenged to find a solution to fix this undesired geometry.
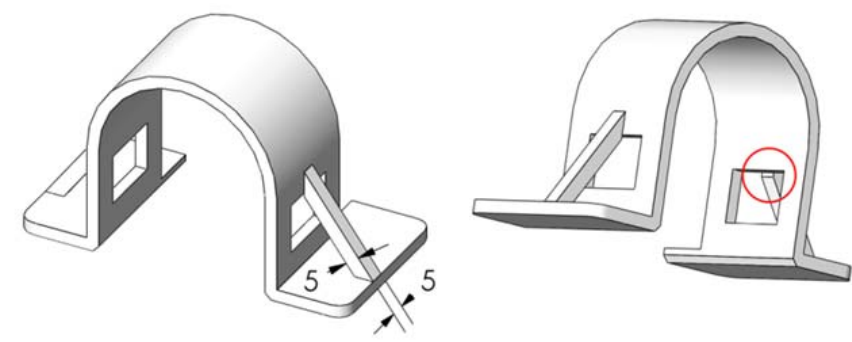

Figure 7. INITIAL ALTERATION (LEFT) CAUSES UNWANTED EFFECT (RED CIRCLE)

We identified three major modeling approaches to solve this problem: increasing the angle of the ribs, reducing the dimensions of the square cuts on both sides of the part, or changing the position of the side cuts by moving them closer to the bottom (see Figure 10). 


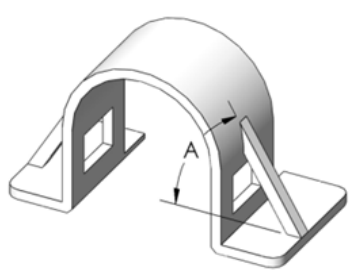

Solution 1: Increase rib angle

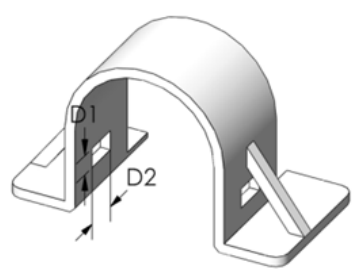

Solution 2: Change side cut dimensions

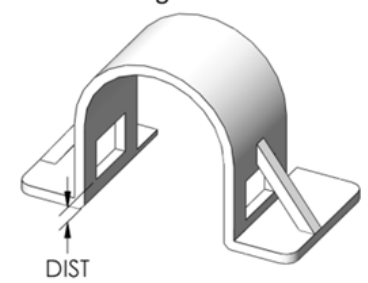

Solution 3. Move side cut down

Figure 10. POSSIBLE SOLUTIONS TO DESIGN PROBLEM PRESENTED IN STUDY 2

The information included in the annotated model contains remarks that the original designer of the part considered valuable. This knowledge explicitly suggests maintaining the current rib angle (because results of previous analysis confirmed optimum angle, for example) and the dimensions of the side cut (perhaps because another component must fit through these cuts). Regardless of reasons, these restrictions and design requirements will certainly determine how the model must be altered and what design conditions need to be met at all times (e.g. the size of the side cuts and the rib angle). Therefore, according to the annotations, modifying the position of the side cuts (solution 3) is the most effective approach. Based on this information, we hypothesized that when critical design decisions must be made, annotated models can help designers select the most appropriate modeling procedure when multiple possibilities are available. We define the modeling approach as the independent categorical variable $\mathrm{X}_{2}$, with values:

Table 4. VALUES OF $\mathrm{X}_{2}$

\begin{tabular}{|c|c|}
\hline Values of $X_{2}$ & Description of the Modeling Approach \\
\hline Solution 1 (S1) & Increase rib angle \\
\hline Solution 2 (S2) & Modify cut dimensions \\
\hline Solution 3 (S3) & Displace side cut \\
\hline Solution 4 (S4) & Other approaches \\
\hline
\end{tabular}

“Solution 4: Other approaches" represents incorrect modeling procedures, such as adding new geometry to the cut as a new feature (inefficient), or incorrectly trimming the size of the rib (the rib must remain at $5 \mathrm{~mm}$ x $5 \mathrm{~mm}$ ). The results of our study are shown in Table 5 and Figures 11 and 12. Similarly to the previous study, we performed a Chi-Square Test to examine the difference in modeling approaches between the control and experimental groups. Based on the resulting $p$-value $(p<0.001)$, we can conclude that there is a significant difference between the experimental and control groups. When critical design decisions about a model need to be made, annotated models are more valuable than non-annotated models in terms of communicating design requirements.

Table 5. ACTUAL AND EXPECTED VALUES FOR STUDY 2

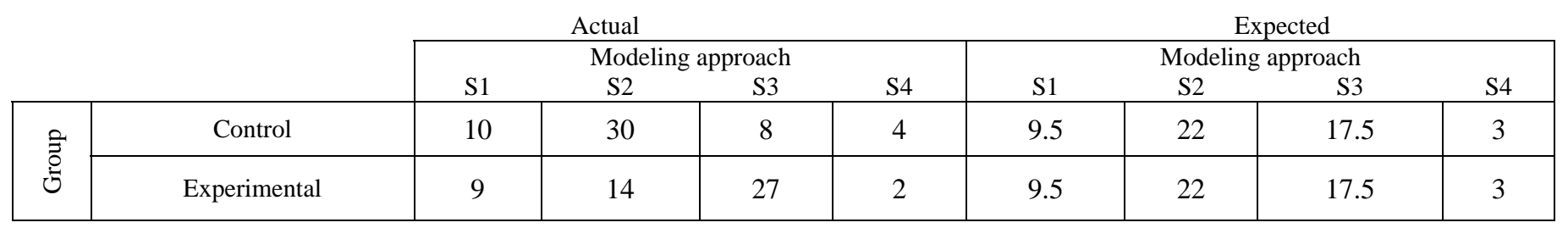



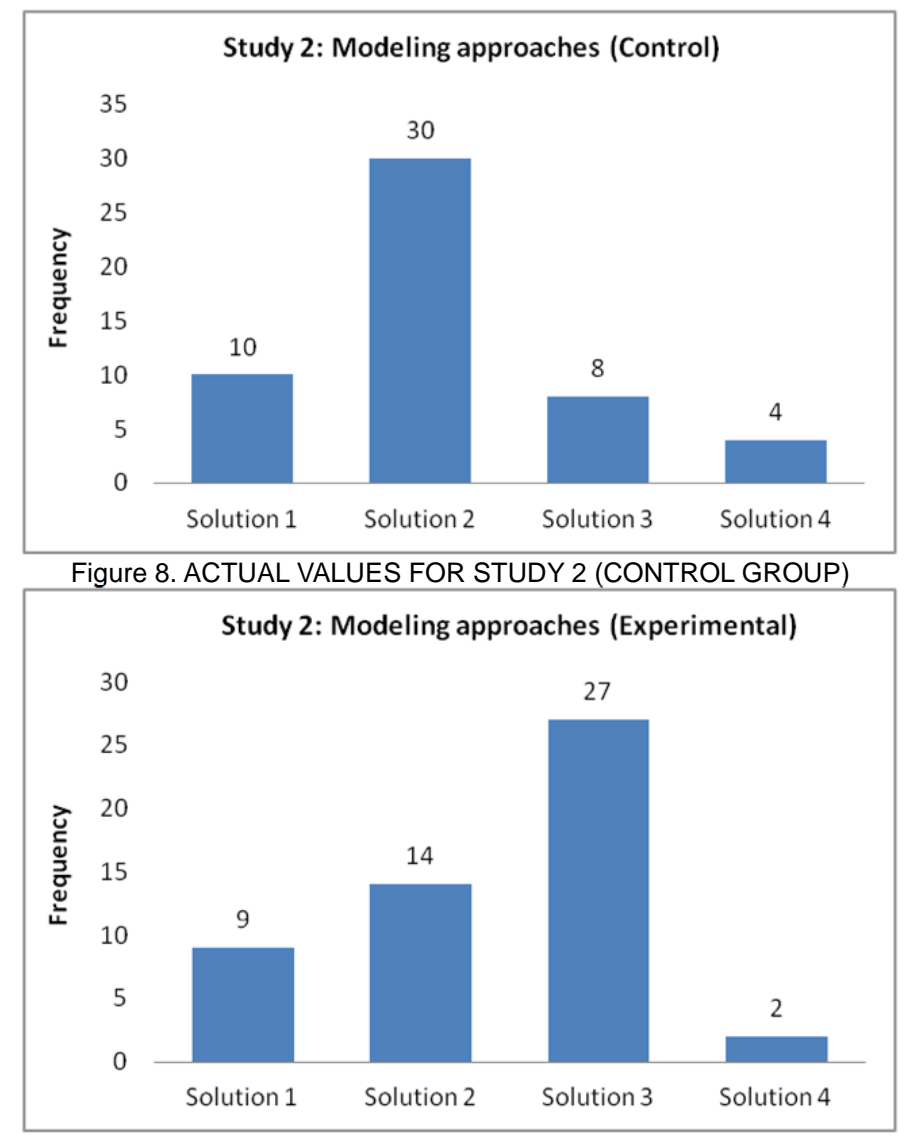

Figure 9. ACTUAL VALUES FOR STUDY 2 (EXPERIMENTAL GROUP)

\section{CONCLUSIONS AND FUTURE WORK}

The role of 3D annotations as carriers of product information (Geometric Dimensioning and Tolerancing, material specifications, etc) within CAD models has been found successful and its use has increased partly because of the formalization of new standards and the popularization of the Model-Based Enterprise paradigm. However, the ability to communicate design intent information using annotations has proven to be challenging, although there are reasons to believe they can serve this purpose effectively as long as proper mechanisms are in place.

Our research is focused on studying the potential of model annotations as design communication elements to facilitate CAD model reuse and alteration, which can improve CAD quality and ultimately impact collaborative and cooperative development.

In this paper, we took a first step towards understanding and evaluating user's response to different CAD challenges when design annotations are present. Our goal was to find evidence of higher quality models (in terms of modeling methodology and model reusability) when annotated models were used. We conducted two experiments: a model alteration study and a design study. In both cases, results show that when users manipulate properly annotated models, even with no prior warning, they select more efficient modeling procedures and create models that are more reusable. Models that have been annotated with design intent information provide a statistically significant value over non-annotated models. For a more comprehensive study, we are preparing a larger and wider variety of models, representing various designs and modeling scenarios.

As future work, we are interested in expanding the scope of the initial experiments reported in this paper. In the short term, we plan to compare user's performance when design intent information is presented as annotations embedded in the CAD model and when it is provided in a different form, such as an additional document or a record on an external repository. Further work is required to evaluate the acceptance and user motivation of expressing design intent using annotations.

Additionally, we intend to study visual clutter and annotation overload effects, which can help determine the optimal number of annotations that can be included in a model without interfering with the modeling process or creating confusion. Finally, it would be interesting to study the effects of writing style and content of the annotations on design intent communication. Determining if users are providing too much information, too little, or simply not expressing the concept correctly can lead to the development of a standardized structure or language for CAD annotations related to design. 


\section{REFERENCES}

[1] Salehi, V., McMahon, C., 2009, "Action Research into the Use of Parametric Associative CAD Systems in an Industrial Context”. In Proceedings of the 17th International Conference on Engineering Design, ICED09, Vol. 5, pp. 133-144.

[2] Toussaint, J., Cheng, K., 2002, “Design Agility and Manufacturing Responsiveness on the Web”. Integrated Manufacturing Systems, 13(5), pp. 328-339.

[3] Chang, K.H., Silva, J., Bryant, I., 1999, "Concurrent Design and Manufacturing for Mechanical Systems". Concurrent Engineering, 7, pp. 290-308.

[4] Jackson, C., Buxton, M., 2007, “The Design Reuse Benchmark Report: Seizing the Opportunity to Shorten Product Development”, Aberdeen Group, Boston.

[5] Lang, S.Y.T., Dickinson, J., Buchal, R.O., 2002, “Cognitive Factors in Distributed Design”. Computers in Industry, 48, pp. 8998.

[6] Demathew, T., 1989, "Productivity by Computer Aided Design”. Automation, 36(1), pp. 52-54.

[7] Robertson, D., Allen, T.J., 1993, “CAD-System Use and Engineering Performance”. IEEE Transactions on Engineering Management, 40(3), pp. 274-282.

[8] Baba, Y., Nobeoka, K., 1998, "Towards Knowledge-Based Product Development: The 3D CAD Model of Knowledge Creation”. Research Policy, 26(6), pp. 643-659.

[9] Teresko, J., 1988, “Speeding the Product Development Cycle”. Industry Week, 237(6), pp. 40-42.

[10] Liu, D.T., Xu, X.W., 2001, “A Review of Web-Based Product Data Management Systems”. Computers in Industry, 44(3), pp. 251-262.

[11] Caldwell, B., Mocko, G.M., 2008, “Product Data Management in Undergraduate Education”. ASME International Conference on Computers and Information in Engineering. Brooklyn, New York, 2008.

[12] Ullman, D.G. 2010. The Mechanical Design Process, $4^{\text {th }}$ ed. Mc Graw Hill, Boston.

[13] Iyer, N., Jayanti, S., Lou, K., Kalyanaraman, Y., Ramani, K., (2005), "Shape-based Searching for Product Lifecycle Applications”. Computer-Aided Design, 37(13), pp. 1435-1446.

[14] Bodein, Y., Rose, B., Caillaud, E., (2013), "A Roadmap for Parametric CAD Efficiency in the Automotive Industry". Computer-Aided Design, 45(10), pp. 1198-1214.

[15] ASME, 2012. ASME Y14.41-2012 Digital Product Definition Data Practices. The American Society of Mechanical Engineers, New York.

[16] ISO, 2006. ISO 16792:2006 Technical Product Documentation - Digital Product Definition Data Practices. Organisation Internationale de Normalisation, Genève, Suisse.

[17] Iyer, G.R., Mills, J.J., 2006, “Design Intent in 2D CAD: Definition and Survey”. Computer-Aided Design and Applications, 3(14), pp. 259-267.

[18] Henderson, M.R., 1993, “Representing Functionality and Design Intent in Product Models”. Proceedings on the second ACM symposium on Solid modeling and Applications, Montreal, Quebec, Canada, pp. 387-396.

[19] Conklin, E.J., Yakemovic, K.C., 1991, “A Process Oriented Approach to Design Rationale”. Human-Computer Interaction, 6, pp 357-391.

[20] Lee, J., Lai, K., 1991, “What’s in design rationale” Human-Computer Interaction, 6(3-4), pp. 251-280.

[21] Hounsell, M.S., Case, K., 1997, “Morphological and Volumetrical Feature-Based Designer’s Intents”. Proceedings of the 13th National Conference on Manufacturing Research. Glasgow, Scotland, pp. 64-68.

[22] Horvath, L., Rudas, I.J., 2003, “Modeling Behavior of Engineering Objects Using Design Intent Model” Industrial Electronics Society, 2003. IECON'03. The 29th Annual Conference of the IEEE, 1, pp. 872-876.

[23] Nielsen, E., Dixon, J., Zinsmeister, G., 1991, “Capturing and Using Designer Intent in a Design-With-Features System" Proceedings Design Theory and Methodology, DE, vol. 31, pp. 95-102.

[24] Brissaud, D., Garro, O., Poveda, O., 2003, “Design Process Rationale Capture and Support by Abstraction of Criteria”. Research in Engineering Design, 14, pp. 162-172.

[25] Pena-Mora, F., Sriram, D., Logcher, R., 1993, “SHARED-DRIMS: SHARED Design Recommendation-Intent Management System”. Proceedings of the Second Workshop Enabling Technologies: Infrastructure for Collaborative Enterprises, pp. 213221.

[26] Ding, L., Davies, D., McMahon, C., 2008, “Sharing Information throughout a Product Lifecycle via Markup of Product Models”. Proceedings of the ASME International Design Engineering Technical Conferences \& Computers and Information in Engineering Conference, vol. 3, pp. 1267-1275.

[27] Sandberg, S., Näsström, M., 2007, “A Proposed Method to Preserve Knowledge and Information by Use of Knowledge Enabled Engineering”. Proceeding of the ASME International Design Engineering Technical Conferences \& Computers and Information in Engineering Conference, Las Vegas, Nevada, USA, vol. 2, pp. 207-212.

[28] Alducin-Quintero, G., Rojo, A., Plata, F., Hernández, A., Contero, M., 2012, “3D Model Annotation as a Tool for Improving Design Intent Communication: A Case Study on its Impact in the Engineering Change Process”. Proceedings of the ASME International Design Engineering Technical Conferences \& Computers and Information in Engineering Conference, Chicago, Illinois, USA, vol. 2, pp. 349-356. 
[29] Dorribo-Camba, J., Alducin-Quintero, G, Perona, P., Contero, M., 2013, "Enhancing Model Reuse through 3D Annotations: A Theoretical Proposal for an Annotation-Centered Design Intent and Design Rationale Communication”. Proceedings of the ASME International Mechanical Engineering Congress \& Exposition, San Diego, CA, USA. Paper No. IMECE2013-64595.

[30] Ding, L., Ball, A., Patel, M., Matthews, J., Mullineux, G., 2009, “Strategies for the Collaborative Use of CAD Product Models”. Proceedings of the 17th International Conference on Engineering Design, ICED09, 8, pp. 123-134.

[31] Bracewell, R.H., Wallace, K.M., 2003, “A Tool for Capturing Design Rationale”. Proceedings of the 14th International Conference on Engineering Design, ICED’03. Design Society, Stockholm, Sweden, pp. 185-186.

[32] Boujut, J.F., Dugdale, J., 2006, “Design of a 3D Annotation Tool for Supporting Evaluation Activities in Engineering Design”. Cooperative Systems Design, COOP 6, pp.1-8.

[33] Davies D., McMahon C.A., 2006, “Multiple Viewpoint Design Modelling through Semantic Markup”. Proceedings of ASME 2006 International Design Engineering Technical Conferences and Computers and Information in Engineering Conference, Philadelphia, Pennsylvania, USA, vol. 3, pp. 561-571.

[34] Patel, M., Ball, A., Ding, L., 2008, “Curation and Preservation of CAD Engineering Models in Product Lifecycle Management”. Conference on Virtual Systems and Multimedia Dedicated to Digital Heritage (VSMM’08), University of Bath, pp. 59-66.

[35] Li, C., McMahon, C., Newnes, L., 2009, “Annotation in Product Lifecycle Management: A Review of Approaches". Proceedings of the ASME International Design Engineering Technical Conferences and Computers and Information in Engineering Conference, vol. 2, pp. 797-806. 